Preference is given to letters commenting on contributions published recently in the JRSM. They should not exceed 300 words and should be typed double spaced

\section{Lack of confidentiality with the Picture Archiving and Communication System (PACS)}

Within a few months after the introduction of PACS in our trust for viewing and storing radiology images we noticed two very distinctive images being accessed recurrently in our department. These were accessed mainly by staff from other departments who were demonstrating the abnormalities to groups of onlookers. The first image is of a priapic penis. The second shows a massive vibrator in a patient's colon. Both the images have had measurements taken and the virtual rulers have been left across the images.

Our NHS trust extends over two separate hospital sites. The second patient never attended our hospital site; he was treated at the adjacent site. Both these images are freely available for viewing across both sites by means of PACS. It has become apparent that the names of these patients are known to a wide variety of medical, nursing and ancillary staff from various specialties across the two hospital sites.

From access records obtained by our information technology department we found, for the three PACS terminals in our emergency department (ED), the first patient's images had been viewed on 9 instances by 5 different users, whilst the second patient's images had been viewed on 16 instances by 10 different users. These figures are an underestimation of the number of times these images are downloaded as the PACS system is also aligned to the world-wide-web browser; web-based viewings cannot be counted and are untraceable to user.

We took several steps to decrease the chance of recurrence. Notices were put on all the computer terminals reminding users to log out after each episode. We informed all users of the terminals that we were able electronically to ascertain what images they were accessing. The doctors who were known to have downloaded these images in the ED were asked by a consultant whether they believed they were acting in the best interests of patient care and whether they believed they were upholding the patient's confidentiality. They were reminded of the Caldicott principles. ${ }^{1}$ Since these procedures have been put into place only one similar episode has occurred to our knowledge.

The advantages of PACS and similar systems are ease of access and immediate sharing of data and images that can result in better patient care. ${ }^{2}$ With an electronic system, images can be viewed trust-wide, on any terminal, at any time. From the initial impulse to download an image to actually looking at the image takes only a few seconds; if you remember the name you can find the image. With hard copies, the effort involved in finding the radiograph gives time for the clinician to reflect whether there is a need to share the information; hard copies can only be in one place at any one time. They tend to stay with the patient and the team looking after the patient, and are filed away when the patient is discharged.

Some work has been published on the confidentiality of computer-stored medical information. ${ }^{3}$ With specific reference to radiographs stored on a web-based system such as PACS much effort has gone into ensuring that the information is secure. In relation to the instances at our trust illustrated above, we find that simply using passwords and usernames to access medical information may not be enough. The mere knowledge of a patient's name is sufficient information to enable anyone logged onto the system to access all imaging for that patient. With the advent of systems to make more medical information available this worry becomes increasingly pertinent across other fields.

Medical information should be handled discreetly and in such a way that patient information is fully protected. ${ }^{4}$ The Caldicott Report, ${ }^{1}$ published in 1997, made several recommendations aimed at protecting such information:

Justify the purpose(s) of using confidential information Only use it when absolutely necessary

Use the minimum that is required

Access should be on a strictly need-to-know basis

Everyone must understand his or her responsibilities

Understand and comply with the law.

Nothing can guard against the voyeuristic nature of human beings. However, the use of information technology and digital web-based information systems being brought into use nationally throughout all NHS trusts ${ }^{5,6}$ brings into question how to ensure that patients' personal information is accessed only by those who 'need to know', with utmost respect for confidentiality.

\section{Names and addresses supplied*}

\section{REFERENCES}

1 Department of Health. The Caldicott Committee - Report on the Review of Patient Identifiable Information. London: DoH, 1997 [http:// www.publications.doh.gov.uk/ipu/confiden/report/caldrep.pdf]

2 Muller H, Michoux N, Bandon D, Geissbuhler A. A review of contentbased image retrieval systems in medical applications - clinical benefits and future directions. Int J Med Inf 2004;73:1-23

3 Hodges JG Jr, Gostin LO, Jacobsen PD. Legal issues concerning electronic health information: privacy quality and liability JAMA 1988; 282: $1466-71$

4 Department of Health. Confidentiality - NHS Code of Practice. Final Report London: DoH, 2003 [http://www.dh.gov.uk/assetRoot/04/06/92/ 54/04069254.pdf]

5 Wanless D. Securing our Future Health: Taking a Long Term View. London: HM Treasury, 2002 [http://www.hm-treasury.gov.uk/ Consultations_and_Legislation/wanless/consult_wanless_final.cfm]

6 Department of Health press release. New electronic X-rays and scans will mean faster diagnosis for patients. 20 May 2004 [http:// www.dh.gov.uk/assetRoot/04/08/20/77/04082077.pdf]

*The JRSM waived its usual rule against anonymity because the authors wished to make a general point without embarrassing local colleagues or their hospital trust-Editor 


\section{Specialist medical abbreviations as a foreign language}

In journals, unexplained abbreviations cause frustration and confusion, ${ }^{1,2}$ and the same is likely to be true of case notes from an unfamiliar specialty. Serious drug errors have arisen from misunderstanding of abbreviations., ${ }^{3,4}$ With the changes in medical working hours in the UK, it is now commonplace for a doctor to cover several different subspecialties. We therefore designed a questionnaire to assess how well some abbreviations widely used in ear, nose and throat surgery (ENT) were understood by junior doctors in other specialties. The thirteen chosen abbreviations, selected from those previously judged 'acceptable' in operation notes, ${ }^{5}$ were as follows: TA\& $\mathrm{G}=$ tonsils, adenoids and grommets; $\mathrm{SMR}=$ submucous resection; $\mathrm{SMD}=$ submucous diathermy; DIT=diathermy of inferior turbinate; FESS=functional endoscopic sinus surgery; MUA=manipulation under anaesthesia; PNS=postnasal space; EUA= examination under anaesthesia; $\mathrm{OE}=$ otitis externa; $\mathrm{CSOM}=$ chronic suppurative otitis media; $\mathrm{OME}=$ otitis media with effusion; $\mathrm{TM}=$ tympanic membrane; $\mathrm{FB}=$ foreign body. For each of the abbreviations, the questionnaire gave a hint of the context - for example, 'name of operation' or 'diagnosis'. Participants were also asked for reactions (from $1=$ strongly disagree to $5=$ strongly agree) to the statement that 'any doctor should be able to read and understand notes from any specialty'. The questionnaire was distributed to all junior doctors attending lunchtime meetings at two different hospitals (no duplication) - namely, 4 specialist registrars, 20 senior house officers and 21 preregistration house officers, in specialties including accident and emergency, orthopaedics, general medicine and general surgery; none had held a post in ENT. Answers about abbreviations were scored as either correct or incorrect, though with some latitude if close enough for the clinical sense to be preserved. The response rate was $100 \%$, and Figure 1 shows the rates of correct answers. There was a tendency to agree with the proposal that doctors should be able to understand notes from any specialty (median score 4).

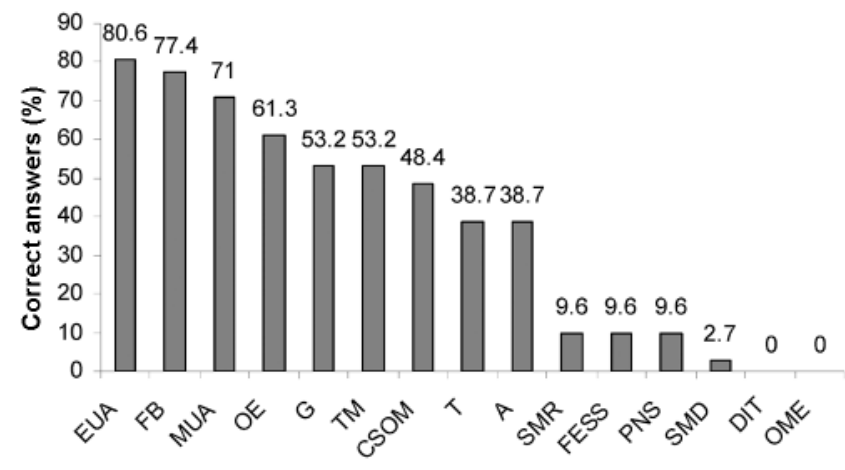

This small study indicates that 6 of 13 commonly used abbreviations in ENT were unclear to more than $90 \%$ of junior doctors from other specialties. With the European Working Time Directive, cross-cover will be an increasing feature of life in the National Health Service; and, if abbreviations continue to be used in the traditional manner, medical notes may be incompletely understood by the doctor on call. The solution is either to ban abbreviations from medical notes or to distribute widely a list of those acceptable, with explanations.

\section{Prodip Das-Purkayastha}

\section{Katie McLeod}

\section{Richard Canter}

Department of Ear, Nose and Throat, Royal United Hospital, Combe Park, Bath BA1 3NG, UK

Correspondence to: Mr Prodip Das, 12 Beckerley Lane, Holt BA14 6QQ, UK E-mail: prodipdas@hotmail.com

\section{REFERENCES}

1 Wells W. Increasing use of abbreviations is unacceptable. BMJ 2001;322:495

2 Lader EW. Acronym mania. Lancet 2002;360:576

3 Anonymous. Medication errors related to potentially dangerous abbreviations. Sentinel Event Alert 2001;23:1-4

4 Lilley L, Guanci R. Look-alike abbreviations: prescriptions for confusion. Am J Nurs 1997;97:12

5 Bateman ND, Carney S, Gibbin KP An audit of the quality of operation notes in an otolaryngology unit. $J$ R Coll Surg Edinb 1999;44:94-5

\section{Hope and despair}

Dr Sleight's valuable article on voluntary euthanasia (July $2004 J S M^{1}$ ) begins with a misattribution. Jerome Kern wrote the music for the wonderful song 'Ol' Man River' for Showboat, but he did not write words. Credit for the lyric, and so for the quote 'Ah'm tired of liv-in' An' skeered of dying',' should go to Oscar Hammerstein II.

\section{John Peniket}

11 The Avenue, Churchdown,

Gloucester GL3 2HB, UK

\section{REFERENCE}

1 Sleight V. Hope and despair. J R Soc Med 2004;97:354

\section{Death by footwear}

Dr Greenberg (July 2004 JRSM ${ }^{1}$ ) seeks an explanation for a cluster of deaths registered between 1894 and 1900 due to footwear. One possible theory is the rupture of a saccular popliteal aneurysm due to pressure trauma from tight kneelength boots worn by the cavalry and fashionable society. An example of the former may be found in a post mortem pot in the Gordon Museum at Guy's. 
Thomson and Miles' Manual of Surgery published in 1909 mentions a further cause of popliteal aneurysms previously very common in post boys - the repeated flexion and extension of the knee in riding. ${ }^{2}$

Time will tell if current shoe trends will harm anything other than the wallet.

\section{Peter Perkins}

Southbourne Surgery, 17 Beaufort Road, Southbourne, Bournemouth, Dorset BH6 5BF, UK

E-mail: postmaster@gp-J81059.nhs.uk

\section{REFERENCES}

1 Greenberg M. A cluster of deaths by footwear, 1894-1900. J R Soc Med 2004;97:360

2 Thomson A, Miles A. A Manual of Surgery, Vol. 1. London: Henry Frowde and Hodder \& Stoughton, 1909:322

\section{Tuberculosis sanatoria regimens in the 1940s}

The personal diary describing a patient's life in a British TB sanatorium in the 1940s reproduced in Raymond Hurt's article (July $2004 J R S M^{1}$ ) reminded me of Betty Macdonald's quite similar experiences in Seattle, Washington, recalled in her much neglected The Plague and $I .^{2}$ Macdonald was also a patient in the 1940s and although quite different in temperament from Hurt's protagonist, she also was around thirty when first admitted, was the mother of two small children and was fortunate in having a supportive family. Also like Hurt's diarist, she gives a blowby-blow account of clinical practice at the time. Macdonald's book, however, also contains rich descriptions of the psychosocial histories of the sanatorium's patients and staff as well as providing insight into the difficult adjustments such patients experienced upon their return to the world. Adjustments even more vividly described by Elizabeth Simpson in her account of recovery from tuberculous meningitis some thirty years later. ${ }^{3}$

These patients' stories deserve to be remembered not merely for what they teach us of history, but perhaps more importantly for what they say about the ways we all deal with recurrent illness.

Ross Kessel

Limecroft, Shute Hill, Malborough, Devon TQ7 3SF, UK

\section{REFERENCES}

1 Hurt R. Tuberculosis sanatorium regimen in the 1940s: a patient's personal diary. J R Soc Med 2004;97:350-3

2 Macdonald B. The Plague and I. London: Hammond, Hammond, 1948

3 Simpson EL. Notes on an Emergency: A Journal of Recovery. New York: Norton, 1982

\section{Basic training in ophthalmology}

The paper by Professor Fielder and his colleagues (April $2004 J_{R S M}^{1}$ ) provides information that confirms the experience of the Training Committee of the Royal College of Ophthalmologists from our programme of hospital inspections. The issue is complex, and even within a hospital that is well disposed towards surgical training for senior house officers (SHOs) the experience can vary within any given year depending upon the SHOs in post. In general terms, around half of the units we inspect provide reasonable training to their trainees at the time of inspection. The bulk of the remaining units are capable of providing appropriate training experience if they are pressurized into monitoring the training experience of their SHOs and submitting their returns.

Modernizing Medical Careers offers ophthalmology an opportunity to restructure our training programmes. Ophthalmology will require approximately $50 \%$ of the current number of SHOs if we intend to move towards a run-through training grade. The principal issue is the service workload being undertaken throughout the UK by the remaining $50 \%$ of SHOs. This is the problem that needs to be addressed. If the number of SHOs in training were reduced, one would anticipate that a better quality of training would be available to this lesser number of trainees. It is also of great importance that consultant trainers are given sufficient time within their working week to engage with trainees and that the culture of the NHS is not driven by service alone.

\section{Stuart Cook}

Chairman, Training Committee,

Royal College of Ophthalmologists,

17 Cornwall Terrace, London NW1 4QW, UK

\section{REFERENCE}

1 Watson MP, Boulton MG, Gibson A, Murray PI, Moseley MJ, Fielder AR. The state of basic surgical training in the UK: ophthalmology as a case example. J R Soc Med 2004;97:174-8

\section{The well-managed waiting list}

'Treatment delayed is treatment denied'

David Williams ${ }^{1}$

The editorial by Professor Black (April 2004 JRSM$^{2}$ ) extols both the inevitability as well as the virtues of the surgical waiting list. Having practised on both sides of the Atlantic, I find his arguments hard to accept.

A surgical 'waiting list' is a queue activated by discrepancy between the need for services and the operating facilities available, such as a limited number of operating rooms and/or personnel, and at the same time, a plethora 
of patients in need of surgery. Such a queue may be bypassed only by the urgent nature of the existing clinical condition of some of the patients; the others will be served in the order by which they have accessed the system.

The basic characteristic of such a waiting list is that, given a stable number of the populace serviced by that particular institution and occurrence of the conditions requiring surgery, on the one hand, and the 'fixed' narrowness of the 'bottleneck', on the other, the list must grow. For example, if an average of 100 new patients per month is in need of coronary surgery, the hospital can handle only 80 and the waiting list already has 200 patients, then this list has to increase by an average of 20 patients per month and double in ten months.

Does this happen? Not necessarily. Visiting numerous institutions in Europe, I found that in most institutions the list remained steady and the time the patients had to stand in line for surgery remained the same year after year. There were several possible explanations for this. One is that the institution managed to satisfy the ongoing demand but was not able to take care of the already existing waiting list. This is possible but unlikely. Another is that it was 'selfregulating' by attrition. Some patients got tired of waiting and simply got off the list while others sought help at some other, usually private, institution. Some patients whose condition suddenly worsened died while awaiting surgical intervention. Finally, it is indeed possible that the waiting list was artificially maintained, not because of lack of resources, but because the healthcare providers had no intention to make it disappear.

Black indeed lists some of these 'commendable reasons' for the existence of such a 'well-managed' waiting list:

- 'It maximizes efficiency by ensuring steady demand for previous resources, such as staff, theatres and beds' true indeed

- Enhances staff satisfaction and morale by ensuring theatre lists have an interesting mix of cases, and enable training needs to be met - a very weak argument
- 'For some diseases . . . waiting for surgery provides a period of spontaneous resolution of symptoms, therefore avoidance of unnecessary surgery'-true unless it gets worse or the patient dies

- 'And for those whose condition does not improve, it allows time to either reconsider their decision, or prepare both practically and psychologically for their operation'-for 3-6 months?

To these 'benefits' of the waiting list system we may add what is an open secret: for many underfunded health institutions the only way to obtain additional budgeting is to have its request justified by a few hundred indignant patients standing in a queue awaiting surgery.

Even if some of the above arguments are valid one cannot help noticing that none serve the interest of the patient, only the needs of caregivers. That fact is well reflected in Black's statement that the 'principal reason people opt for private care is to avoid excessive waiting'. The fact that people are willing to stand in a queue does not mean that they like it. They may accept it because they believe that it is an economic necessity. The harmful effects of surgical waiting lists are not limited to inconvenience but include psychological and physiological harm - especially in the patient population in the queue for diagnostic and therapeutic cardiac interventions. I cannot accept Black's recommendation that 'payers should focus on the number of patients treated rather than the number of patients awaiting treatment'. There is no such thing as a wellmanaged waiting list - only a poorly managed flow of human beings, standing in line, awaiting care.

Francis Robicsek

Carolinas Heart Institute, 1001 Blythe Blvd, Suite 300, Charlotte, NC 28203, USA E-mail: frobicsek@sanger-clinic.com

\section{REFERENCES}

1 Williams DO. Treatment delayed is treatment denied. Circulation 2004;109:1806-8

2 Black N. Surgical waiting lists are inevitable: time to focus on work undertaken. J R Soc Med 2004;97:159-60

\section{CORRECTION}

Needle-stick injuries in the National Health Service: a culture of silence

This paper (July $2004 J R S M^{1}$ ) should have acknowledged the help of the Clinical Audit Department at East and North Herts NHS Trust, Lister Hospital, and in particular that of Heather Davies and Carol Leckenby.

\section{REFERENCE}

1 Elmiyeh B, Whitaker IS, James MJ, Chahal CAA, Galea A, Alshafi K. Needle-stick injuries in the National Health Service: a culture of silence. J R Soc Med 2004;97:326-7 